AperTO - Archivio Istituzionale Open Access dell'Università di Torino

\title{
On the mechanochemical activation by ultrasound
}

\section{This is the author's manuscript}

Original Citation:

Availability:

This version is available http://hdl.handle.net/2318/143047

since 2016-02-02T12:08:22Z

Published version:

DOI:10.1039/C2CS35456J

Terms of use:

Open Access

Anyone can freely access the full text of works made available as "Open Access". Works made available under a Creative Commons license can be used according to the terms and conditions of said license. Use of all other works requires consent of the right holder (author or publisher) if not exempted from copyright protection by the applicable law. 


\section{(3) \\ UNIVERSITÀ DEGLI STUDI DI TORINO}

This is an author version of the contribution published on:

On the mechanochemical activation by ultrasound

Cravotto G.; Calcio Gaudino E. ; Cintas P

Chem. Soc. Rev., 2013,42, 7521-7534

The definitive version is available at:

DOI: $10.1039 / C 2 C S 35456 J$ 


\title{
On the mechanochemical activation by ultrasound
}

Cravotto G.; Calcio Gaudino E. ; Cintas P.

\begin{abstract}
Chemists have discovered, and recently actively exploited, the fact that subjecting certain molecules to ultrasound waves can bring about transformations that give insight into the correlation between classical tribological processes and the mechanical action caused by collapsing microbubbles when sonic waves propagate through a liquid medium. Chemical transformations induced by ultrasound take place in solution via mechanisms that are markedly different from those associated with molecular activation in the solid state. Both fields, however, share some striking similarities and numerous sonochemical reactions can be rationalized in purely mechanical terms. This tutorial review examines the tribochemical interpretation of sonochemical reactivity and how the multifaceted action of cavitational phenomena determines molecular evolution. A series of case studies involving solids, crystals, and polymers illustrate the mechanical properties of sound waves.
\end{abstract}

\section{Introduction: bridging the gap}

There is little doubt that mechanochemistry is a rising star in modern science and the numerous monographs, review articles and themed issues that have appeared in recent years surely attest the fact. ${ }^{1-5}$ For scholars scrutinizing scientific psychology, this renaissance may come as a surprise, as the use of mechanical forces to induce reactivity is the oldest and simplest technique used by mankind and one which has existed since before the dawn of science. Grinding, milling, cutting, polishing, shearing, stirring and scratching provide the driving force for mechanochemical transformations.

Much like in other fields, the lure of simplicity, combined with wide applicability, has invariably led to a literature avalanche that, in this case, has deviated from the conceptual basis of mechanochemistry. This fact has been highlighted by some experts and Kaupp, in particular, has paid considerable attention and effort to illustrate what mechanochemistry actually is. ${ }^{6}$ In a nutshell, it is a branch of solid-state chemistry where intramolecular bonds are broken by mechanical action followed by further chemical reactions. In other words, mechanical energy causes bond breaking as the first step. In this context, mechanochemistry applies to infinitely covalent inorganic crystals, metals, polymers, and small organic molecules with both weak and strong bonds. This definition excludes, however, other solid-state molecular reactions and purely physical activation (mechanophysics; e.g. phase transitions or atomic changes in crystal lattices). Similarly, the reduction of particle size or production of micronized crystallites leading to high surface areas for solidsolid or solid-liquid reactions should not be considered mechanochemistry. Accordingly, mechanochemistry does not apply in a strict sense to liquid or solid-liquid reactions, even though the terms mechanochemical and mechanosynthesis are commonly encountered in numerous examples (vide infra). Obviously, purely mechanical effects can take place in solution and heterogeneous systems, thus accounting for enhanced reactivity. However, the creation of large tensile forces in liquids via simple friction or agitation is not a simple task. Sonication provides a viable exception by virtue of the mechanical and chemical events it causes via the unique phenomenon of acoustic cavitation. In recent years there has been growing interest in exploiting the action of ultrasound waves in numerous domains, which include crystal engineering, materials chemistry and polymer-based reactions, all of which highlight the 
"mechanical activation" niche. There is a certain sense of reductionism in the latter view, although it is true that large and non-volatile substrates are unlikely to withstand the extreme conditions that occur inside the cavitating bubbles. Cavitation is not only a non-linear phenomenon, but it is also dependent on external parameters such as frequency, intensity and the nature of solvents. Collectively these variables influence both the lifetime and evolution of microbubbles, particularly in the vicinity of solids.

Sonochemistry cannot be circumscribed as mechanochemistry; it is likely more appropriate to denote the field as cavitational chemistry, ${ }^{7}$ because there is no doubt that the origin of the sonochemical effect is cavitation. There are basically two classes of effects; radical and mechanical. The former arises from the sonolysis of molecules (i.e. homolytic fragmentation to radical species and/or generation of excited states), which occurs largely in the cavity interior as well as at the bubble interface. Mechanical effects via cavity collapse arise from shear forces, microjets and shock waves that occur outside the bubble, resulting in profound physical changes when solids or metals are present. These changes include improved mass transfer, particle size reduction, surface erosion and cleaning, and are often accompanied by changes in particle properties. In numerous reactions, an interpretation of reactivity requires the mutual action of single-electron transfer (SET) pathways and physical mechanisms, which cannot easily be disentangled, to be considered (sonoelectrochemistry is a paradigmatic case).

Likewise, mechanochemistry can also generate radicals via the breaking of weak bonds and under extreme surface plasma conditions where covalent crystals are cracked by mechanical impact. ${ }^{6}$ Clearly, both scenarios and strategies are different, although goals may be similar. Moreover, as will be discussed later, mechanosyntheses involving milling or grinding of solids may actually occur via a low-melting eutectic phase, and the use of small or catalytic amounts of a liquid (liquid-assisted grinding) has become standard practice among scientists exploring mechanochemical reactions (although they are considered heterodox reactions in terms of the original definition, vide supra). These processes resemble well-known examples of sonochemical activation in liquid-liquid and liquid-solid systems, where the action of waves at the interface plays a crucial role.

This review is aimed at unveiling the features and paths where tribochemistry and sonochemistry meet and highlighting, whenever possible, comparative aspects. The article also focuses on cavitation-based mechanisms, although mechanical effects can be best recognized in low-energy acoustic fields, even in the audible sound range (see the next section) which is a promising area indeed. Researchers and advanced students alike will learn that chemical and physical effects can be attained with waves even without cavitation, which, in itself, is nothing new. As usual we borrow knowledge from our talented predecessors; a fact that reminds us that history is indeed science's best friend.

\section{The energy of sound}

A primer on acoustics may be complex material for most chemists. For the purpose of this article, a little background knowledge will suffice. Sound waves are longitudinal pressure waves, which means that compression takes place in the same direction as the wave itself. It is pertinent to mention that seismic waves generated by earthquakes consist of both primary (compressional or longitudinal in nature) and secondary (shear or transverse) waves. The propagation of sound in a medium constitutes the transmission of mechanical energy. By definition, the intensity of the wave is the average rate of energy transfer per unit time per unit area (also expressed as the average power per unit area). Humans can detect an audible stimulus (hearing) with an average intensity of only $10^{-16} \mathrm{~W} \mathrm{~cm}^{-2} .8$ Sound waves are not necessarily periodic, although those of scientific interest are characterized by frequency and wavelength (i.e. are periodic) and can be expressed in a harmonic equation. In such waves the disturbance (compression or 
rarefaction of the fluid) from a source of sound repeats itself regularly. Most humans will neither hear vibrations at frequencies below $10 \mathrm{~Hz}$ (infrasonic), nor beyond $20 \mathrm{kHz}$ (ultrasonic), even if the intensity is high. These inaudible high-frequency sound waves are periodic stress waves that produce a deformation of the medium through which they propagate.

Applications of ultrasound can be divided into low-energy and high-energy fields. In the former, the amplitude (the height of the wave from its highest point to the lowest point) is low enough to ensure that the medium remains largely unchanged except for a slight increase in temperature. Low-energy ultrasonic waves provide a good amount of information about a material's molecular properties through studies of absorption and relaxation effects, which cannot be treated here.

In stark contrast, high-energy waves irreversibly modify the medium, either by the generation of intense mechanical stress or high localized heating, both of which arise from cavitation. In short, if the ultrasonic pressure exceeds the average pressure in a liquid, the pressure of the latter falls below zero (negative pressure) and the medium fragments into small cavities which expand as the pressure rises and then, as the pressure starts to fall, become unstable and collapse violently. At the end of this collapse, the gas within the cavity is highly compressed and heated (measurements indicate temperatures of at least ca. $4500 \mathrm{~K}$ and pressures of several hundreds of atmospheres). Such extreme conditions are alleviated by the radiation of shock waves along with shear forces that cause physical and chemical effects; mixing liquids that usually would not do so, breaking up cells and macromolecules and triggering chemical reactions. ${ }^{9}$ The cavities (microbubbles created in the liquid for a few microseconds) behave as microreactors where volatile substances may be broken up and then released into the bulk liquid as high-energy species. This scenario is typical of so-called true sonochemistry as the cavitational chemistry actually participates in the reaction outcome. $^{7}$

Mechanical stress caused by acoustic waves had been observed and reported much earlier than the accidental discovery of cavitation at the end of the 19th century. In 1831, Michael Faraday read a paper (plus an appendix) to the Royal Society entitled On a peculiar class of acoustical figures, describing the forms and patterns adopted by some fluids (namely water, milk, egg white, ink and turpentine) in contact with vibrating elastic surfaces. ${ }^{10}$ Faraday observed crispation; a phenomenon described as standing-wave deformations of the fluid surface. Such waves can be observed in numerous systems (Faraday reported this phenomenon in sand and mercury as well) and can take different forms, from fine stripes to regular polygons and even quasiperiodic patterns. Faraday waves complement other processes (BelousovZhabotinsky oscillating reactions and Turing models) accounting for pattern formation and morphogenesis. ${ }^{11}$

The first observation of standing waves in air dates back to the seminal work of August Kundt in $1866 .{ }^{12} \mathrm{~A}$ schematic illustration of his experiment is shown in Fig. 1, which consists of a transparent horizontal pipe containing a small amount of fine powder (cork dust, lycopodium or talc) distributed evenly along the tube. A transducer (sound source) at one end emits acoustic waves and a movable piston at the other is adjusted so that the chamber becomes resonant at that frequency (i.e. the length is a multiple of the wavelength of the sound wave). The resulting standing wave causes the powder to agglomerate at certain points (wave nodes) along the tube. The motion is actually a manifestation of acoustic streaming generated by the interaction of pressure waves with the boundary layer of air at the surface. This observation was also extended to standing waves in liquids, in close resemblance to Faraday waves, ${ }^{13}$ and much later the aggregation mode of different materials under this mechanical action was demonstrated; toluene in water aggregated at pressure nodes while denser quartz particles moved to pressure antinodes. ${ }^{14}$ Standing waves 
had remained a scientific curiosity for decades until the medical observation made in the early 1970s that showed ultrasound stopping the flow of blood in living tissue. This publication in Nature attracted interest and boosted further activity. ${ }^{15}$ Low-power standing waves extending into the $\mathrm{MHz}$ region have found numerous applications in the separation of blood cells and other particles as well as in the design of microfluidic lab-on-a-chip devices. ${ }^{16-18}$

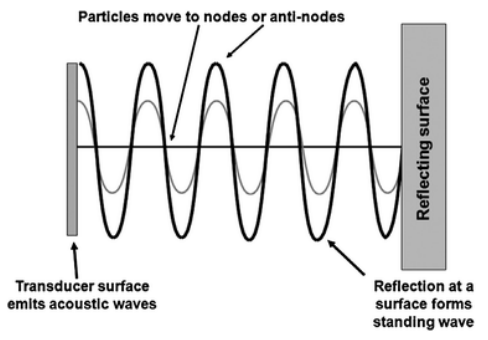

Fig. 1 Formation of standing waves in Kundt's tube. The transducer can be a loudspeaker attached to a generator that produces a sinusoidal wave. The reflecting surface is a movable piston used to adjust the length of the tube. Reprinted from ref. 16 by permission of Elsevier Science B.V.

Standing waves can help with sorting of cells because of the distinctive action of acoustic waves on larger objects relative to smaller ones. Cavitation may occur in standing waves as bubbles concentrate on the antinodes, an effect resulting from Bjerknes forces. However, the appearance of cavitation in standing waves is affected by frequency, intensity and temperature, among other factors. In therapeutic ultrasound employing high-intensity focused waves, cavitating bubbles may also result in cell damage. ${ }^{16}$

At first sight, one might imagine that, in the absence of cavitation, surface acoustic waves (SAWs) would be unable to trigger any transformation involving bond-breaking and -forming reactions. However, it has been recently reported that SAWs are a convenient energy source for drop-scale synthetic chemistry. ${ }^{19} \mathrm{~A} 40 \mu \mathrm{L}$ drop of reaction mixture (typically $30-40 \mathrm{mg}$ ) is deposited onto a piezoelectric substrate (lithium niobate crystal) between two interdigital transducers ( $250 \mathrm{~nm}$ thick Ti-Al electrodes) connected to an amplifier sine wave generator. The SAWs generated ( $20 \mathrm{MHz}, 10 \mathrm{~nm}$ amplitude) will cause the drop to vibrate vigorously as long as the viscosity of the reaction mixture is low, whereas internal streaming is observed in viscous media. On this small scale, SAWs rapidly increase temperature and some selected organic reactions (Scheme 1) have attained complete conversion in less than $30 \mathrm{~min}$. Similar results have been obtained by simply heating the drop-scale reactions to the same temperatures as those in the irradiated experiments, which clearly point to a thermal effect. Such syntheses have also been reported for microwaves and ultrasound on larger scales and higher powers.

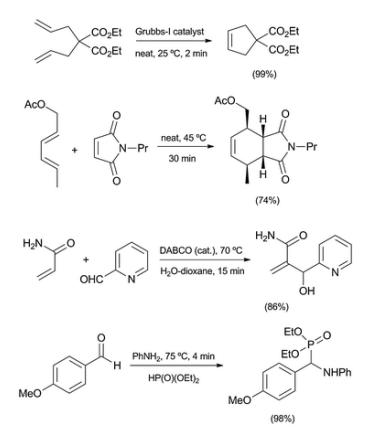

Scheme 1 Results of some selected SAW-irradiated reactions (drop scale). 
According to Luche, who coined the terms true and false sonochemistry, ${ }^{7,20}$ the latter is typical of heterogeneous reactions that follow a polar mechanism and involves non-volatile substrates which are unlikely to undergo molecular fragmentation inside the bubbles. Acceleration results from enhanced mass and energy transfer. This interpretation does not exclude the existence of radical pathways a priori; there are borderline situations where polar and SET mechanisms may occur and sonication usually favours the latter.

The mechanical effects in heterogeneous sonochemistry that arise from cavitation are multiple and effective in increasing chemical reactivity. When bubbles collapse in the vicinity of a surface, the asymmetry of the hydrodynamic flow leads to a liquid jet that is preferentially directed toward the surface. The impacting jet not only causes mechanical damage, but sonoluminescence (SL, i.e. light emission) also results from the vortex ring generated during the asymmetric collapse. The SL may also produce free radicals at the boundary layer and hence in the vicinity of the solid surface (or in large molecules surrounding the bubble), which is a region where sonochemical reactions occur. The second major effect is the intense microstreaming that occurs around bubbles placed on the surface. This microstreaming will dissipate the boundary layer near the bubble and chemical species will be convected rapidly to the surface of a reagent or catalyst. This cavitation-induced microconvection may enhance reactivity still further, even in the absence of radical production. Sonophysicists also remind us of a third, often overlooked, factor that takes place when a bubble collapses near a surface; bubble pulsation, which contributes to microstreaming as well. When flow occurs in the vicinity of a plane boundary, a viscous layer develops that is related to both liquid viscosity and flow velocity. The thickness of the boundary layer is inversely proportional to the square root of the flow rate. At low or moderate velocities, such as those in mechanical stirring, the boundary layer may be a few hundreds of microns thick. Chemical species will hit the surface by diffusion through the layer, a rather slow process. However, as noticed above, acoustic microstreaming will be more efficient in disrupting the layer at the bubble interface. ${ }^{21}$

With the preceding premises, it seems clear that acoustic cavitation may initiate chemical reactions via the action of mechanical pulses, in close similarity to other, solid-state reactions under mechanical loading. This would otherwise be consistent with the initial and broad concept of mechanochemistry by Ostwald, who used the term for the first time. Ostwald simply recognized that the discipline is a part of physical chemistry that is distinctive from thermochemistry, electrochemistry and photochemistry in terms of energy transmission. ${ }^{22}$ Faraday had observed the rapid decomposition and dehydration of some salts under milling and grinding since the early 1820s. However, clear-cut differences in thermal and mechanical activation were only noticed several decades later in the decomposition of $\mathrm{Ag}, \mathrm{Hg}$ and $\mathrm{Au}$ salts, as well as in common displacement reactions. ${ }^{23}$ Likewise, it was reported that the prolonged milling of solids may be inefficient in terms of chemical conversions and only mechanical stress causing surface fusion increased the reaction rate.

These conceptually simple transformations can also be performed under sonication, often using inexpensive cleaning baths, which are suitable for chemical demonstrations. ${ }^{24}$ Thus, copper(II) sulfate pentahydrate, quite stable at room temperature, loses crystallization water upon gentle heating, whereas dehydration occurs under sonication even below ambient temperatures. The rapid reaction of $\mathrm{Mg}$ with cold water, which does not usually occur due to the presence of a MgO coating on the metal surface and the colour change accompanied reaction of copper with sulfur, effectively illustrates the depassivating action of shear forces on surfaces in an ultrasonic field. As mentioned above, this heterogeneous sonochemistry, likely occurring at the bubble-metal interface, may involve the intermediacy of either ions or radicals. 
Copper and dilute hydrochloric acid will not react (due to their electrochemical potentials), but the metal dissolves under sonication and gives rise to a pale blue solution that is characteristic of the $\mathrm{Cu}(\mathrm{II})$ species. ${ }^{24}$

Although the above examples reveal inherent relationships between mechanochemistry and the mechanical action of ultrasonic waves, understanding is hampered by the lack of a unifying rationale. Mechanochemistry can be interpreted using several theories whose modes of excitation and the times of relaxation can vary significantly. ${ }^{23}$ The common criterion is that mechanical stress results from high localized energy; in sonochemistry the gas phase of $\mu \mathrm{m}$-sized bubbles with high temperatures and pressures and very short $(\mu \mathrm{s})$ lifetimes. The current hot-spot model accounts for most experimental observations, while the early electrical theory is unable to reconcile all the facts. ${ }^{9}$ Historically, the first mechanochemical theory introduced in the early 1950 s is referred to the hot-spot model. ${ }^{23,25}$ In short, mechanochemical activation is caused by friction when particles are in contact (surface areas of ca. $1 \mu \mathrm{m}^{2}$ ) for $10^{-4}-10^{-3} \mathrm{~s}$, thereby reaching temperatures over $1000 \mathrm{~K}$ and pressures of several tens of kilobars. These conditions may also be found near the tip of a propagating crack. However, the size of the reactive region, as well as the time during which the extreme thermal effect can exist, will largely be dependent on the material. In any case, the spatio-temporal localization of high energy enables useful comparisons between mechanochemistry and ultrasonic activation to be made; these include the existence of threshold forces, the reduction of induction periods and the creation of dislocations, defects and atomic holes in the early stages which activate the solid, while prolonged action tends to decrease the reactivity because of particle aggregation or sintering as observed in metals. ${ }^{26}$

\section{Ultrasonic treatment of metals}

Traditionally, ultrasonic cavitation at metal surfaces has been of uppermost importance in metal activation, thereby enabling and accelerating the formation of organometallic reagents and metal-assisted couplings. ${ }^{27}$ A decrease in particle size and the creation of fresh metal surfaces appear to be essential ingredients in numerous cases, and account for the well-known facts that air-sensitive lithium or Grignard reagents can be easily generated under mild conditions without an inert atmosphere and in wet solvents. Ultrasonic activation has been extended to other metals in Barbier-type reactions ${ }^{28}$ and azide-alkyne click coupling using metallic copper. ${ }^{29}$

The formation of metal alkyls or aryls via the short milling of the metal in the presence of an organic reagent may also involve the removal of the passivating layer (usually oxide or hydroxide). These transformations do not, however, constitute examples of true mechanochemistry if the reaction proceeds without milling after depassivation (even if acceleration is observed by reducing the induction period). On the other hand, milling may give rise to crystal cracking and resulting surfaces will then be highly reactive as all bonds that previously connected such surfaces lose their neighbouring atoms. Mechanical alloying and formation of intermetallic phases (in general combinations of metals and semimetals of variable stoichiometry) are feasible. ${ }^{6}$

Sonication has recently been applied to the formation of functionalized metal and composite nanomaterials, which can be performed on large scales in industrial processing. These studies provide new insight into the mechanisms of cavitational phenomena near surfaces. ${ }^{30}$ The mechanical damage caused by sonication on metallic plates of $\mathrm{Al}, \mathrm{Ag}, \mathrm{Cu}$ (cubic-face-centered structures) and $\mathrm{Zn}$ (hexagonal lattice) serves as a representative study. ${ }^{31}$ Ultrasound causes micrometric roughness and chemical oxidation, which are usually required to enhance adhesion and further functionalisation. Scanning electron micrographs (SEMs) show that roughness depends on both sample-probe distance (optimal value $=0.5 \mathrm{~mm}$ ) and reaction time. Surface modifications on the Al plate appear rapidly (ca. $1 \mathrm{~min}$ ), although only a few impacts can be 
visualized. Prolonged sonication increases the number of microcraters which act as nucleation zones for cavitation bubbles. Metal grains are detached from the Al surface (10-50 $\mu \mathrm{m}$-sized powder can be collected at the bottom of the reactor), while an aluminium oxide layer develops via the action of hydroxyl radicals arising from water sonolysis. A similar evolution can be observed in other metals (Fig. 2). Copper plates lose the natural oxide layer leading to a reddish colour without a bright lustre. Sonication time needs to be increased to $10 \mathrm{~min}$ to obtain erosion that is comparable to that seen with Al. Silver plates turn white after a few minutes of sonication and significant damage occurs after $10 \mathrm{~min}$. As expected, $\mathrm{Zn}$, a harder metal, is reluctant to undergo surface degradation and grains are locally detached from the surface after $10 \mathrm{~min}$, whereas other zones remain intact. X-ray powder diffraction (XRD) reveals that such superficial changes, as induced by ultrasound, are accompanied by structural changes along the crystalline structure. In Al, a gradual increase in the relative intensity of the peak corresponding to the (111) plane along with the appearance of the peak attributable to the (222) plane is observed. Such XRD patterns are observed at the initial stages of sonication, although no changes occur after $10 \mathrm{~min}$, probably due to cavitational erosion. The same structural modification occurs in $\mathrm{Ag}$ and $\mathrm{Cu}$ as the (111) plane corresponds to the plane of maximum density for a cfc structure and is perpendicular to the ultrasonic beam. Metal grains located at different planes are extracted first. In $\mathrm{Zn}$, the effect is observed in its (001) plane of maximum density for a hexagonal structure.

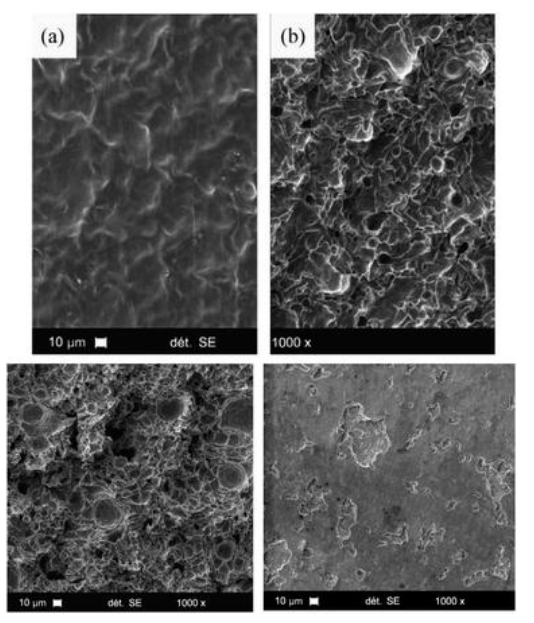

Fig. 2 Top: SEM images of Cu surfaces after ultrasonic treatment for $5 \mathrm{~min}$ (a) and $10 \mathrm{~min}$ (b). Bottom: SEM images of Ag (left) and Zn (right) plates after 10 min sonication. In all cases the sample-probe distance was $0.5 \mathrm{~mm}$. Reproduced from ref. 31 by permission of Elsevier Science B.V.

A further study on elucidating thermal and ultrasonic effects on Al surfaces provides additional mechanistic insight. ${ }^{32}$ The formation of a mesoporous surface is observed in warm water, although ultrasound has little effect when compared to purely thermal treatments. Moreover, the rate of surface change decreases, presumably due to inhomogeneities in the solid-liquid interface under ultrasonic agitation. No significant physicochemical differences between the treatments can be inferred, thus leading to the conclusion that a thermal mechanism preferentially occurs during the sonication of Al plates. A markedly different pathway emerges when metal powders are subjected to ultrasound. SEM and XRD analyses reveal that at low temperature $\left(37^{\circ} \mathrm{C}\right)$, not only is porous morphology visible but the crystal growth of a bayerite phase is also observed. This effect can also be detected in the absence of ultrasound, but at a higher temperature (65 ${ }^{\circ} \mathrm{C}$ ). At this temperature, sonication accelerates the transformation still further and the entire surface is covered by bayerite crystallites and boehmite, although to a lesser extent in the case of the latter. The immobilization of Al powder on a solid surface stops crystal phase change. This suggests that mechanical 
stirring and high-speed motion in the acoustic field modify the processes of dissolution and re-precipitation of metal species.

The sonochemical formation of mesoporous metal surfaces leading to metal sponges with catalytic activity ties in with the putative action of ultrasound at solid-liquid interfaces, as mentioned above (Fig. 3). ${ }^{33}$ The first step involves the action of liquid jets on the metal surface followed by chemical oxidation caused by the highly reactive species that come from solvent sonolysis. The result of this is a porous surface morphology, which is stabilized by a new oxide layer and all-round roughness increases. The process is largely dependent on metal properties and sonochemical exposure conditions. Low-melting point metals ( $\mathrm{Zn}, \mathrm{Mg}$ ) undergo faster modifications than $\mathrm{Al}$, Fe and noble metals. In fact, the latter do not form spongelike structures at all. Ultrasound intensity is also a key factor; the formation of nanosponge morphologies does not take place at $30 \mathrm{~W} \mathrm{~cm}^{-2}$ as revealed by SEM and TEM (transmission electron micrograph) analyses. At $57 \mathrm{~W} \mathrm{~cm}^{-2}$, however, a porous layer appears on top of $\mathrm{Mg}$ and Al plates.

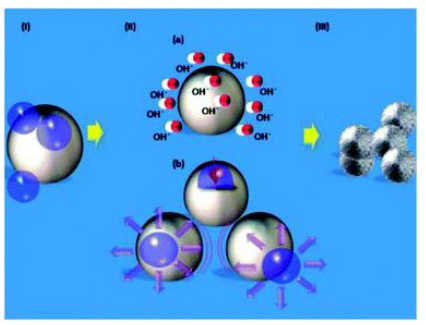

Fig. 3 Schematic formation of mesoporous metals by ultrasonic irradiation; metal particles (I) undergo mechanical and chemical damage after the implosion of cavitation bubbles (II), thus leading to porous structures (III). Reproduced from ref. 33 by permission of the Royal Society of Chemistry.

The importance of radical species in structure modification during mechanical impact has been proven by conducting sonication in an ionic liquid. Due to the low or negligible volatility of such solvents, cavitation is difficult to achieve and the formation of free radicals by homolytic cleavage inside the bubbles is largely suppressed. As a consequence, there is a drastic decrease in the presence of porous morphologies.

Metal alloys (e.g. Al-Ni, Al-Cu-Pd, Al-Pd or Al-Co-Fe) can also be employed in the production of nanocomposites under sonication. The metal component with the lowest melting point creates a mesoporous matrix containing the other metal particles in a homogeneous distribution. Thus, aqueous dispersions subjected to high-intensity ultrasound lead to the formation of porous structures where ultrasound-resistant metal particles ( $\mathrm{Ni}, \mathrm{Pd}$ or $\mathrm{Cu}$ ) are phase segregated and distributed along the matrix. Likewise, nanocomposite materials can be generated via the sonication of $\mathrm{Al}$ or $\mathrm{Mg}$ particles in the presence of noble metal colloids (Fig. 4).

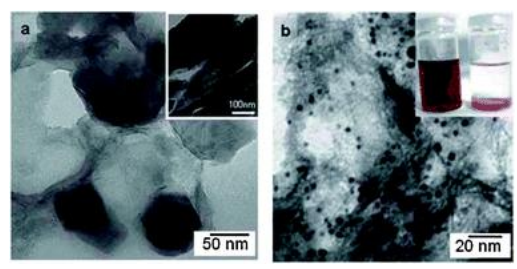

Fig. 4 (a) TEM image of a mesoporous material generated by the sonication of an Al-Ni alloy; the inset shows the untreated alloy. (b) TEM image of Au intercalation inside the aluminium matrix; the inset shows the solution containing mesoporous Al particles and Au nanoparticles before and after intercalation. Reproduced from ref. 33 by permission of the Royal Society of Chemistry. 
Similar intercalation experiments that see Au nanoparticles in mesoporous $\mathrm{TiO}_{2}$ or porous carbon have also been performed under sonication. ${ }^{34,35}$ Again, we are presented with the juxtaposition of the mechanical and chemical effects associated with cavitation. TEM data and theoretical calculations point to the existence of Au atoms on the surface with low coordination numbers. ${ }^{35}$ The existence of coordinatively unsaturated atoms creates sites for further reactions and catalytic activity, and plays a role much like free radicals in organic reactions.

\section{Nucleation and crystal engineering}

The application of power ultrasound to crystallization experiments, i.e. sonocrystallization, constitutes another emerging area in which acoustic vibration has proven to be superior to conventional crystallization methods in numerous aspects; both in reducing nucleation periods and controlling crystal size. ${ }^{36}$ Sonocrystallization has already found a niche in the pharmaceutical industry and has led to crystallization improvements in numerous intermediates and active pharmaceutical ingredients (APIs).

Ultrasound influences crystallization through mechanical effects provided by cavitation and acoustic streaming. These result in a series of benefits, such as sononucleation at lower levels of saturation, often eliminating the need for seed crystals, and the generation of crystals that are smaller and more uniform than those under non-irradiated conditions. The latter is illustrated by the insonated crystallization of adipic acid after prolonged irradiation (Fig. 5). ${ }^{37}$

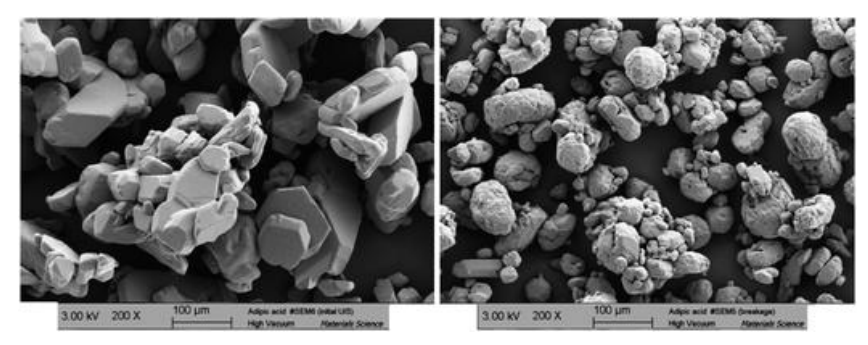

Fig. 5 SEM micrographs of adipic acid crystals: original and irregular particles (left) and round-shaped crystals obtained after 90 min-irradiation at $20 \mathrm{kHz}$ (right). Reproduced from ref. 37 by permission of the American Chemical Society.

The conditions provided by cavitational collapse in solution are obviously different from the mechanochemistry of crystals and solids when weak or strong bonds are mechanically cracked by milling, shearing and shock waves. With exceptions, these transformations are not easy and often require prolonged milling and high temperatures and even pressures in the GPa range. ${ }^{6}$ The co-milling of solids may produce new crystalline materials and cocrystals and is an active research field with implications in the design of novel solid-state structures and pharmaceutical formulations. ${ }^{38,39}$ Most reactions call for liquidassisted grinding, and reactions proceed when the mixture turns sticky. Despite usually being claimed as mechanochemistry, it is always convenient to consider whether the mechanical breaking of bonds precedes the transformation (genuine mechanochemistry) or whether the formation of slurries allows for molecular interactions, which is more akin to false sonochemistry.

A clear-cut advantage to sonocrystallization can be found in the narrow metastable zone (MZ) associated with it. This can be defined as the temperature drop below the solubility curve at which crystals start to separate spontaneously. In the case of molecules that are difficult to crystallize, such as sugars or amino acids, the MZ width can be narrowed by tens of degrees and crystallization can accordingly be observed at lower levels of supersaturation. This effect is also visible when crystallization requires the addition of an 
antisolvent, thereby producing high supersaturation levels. Ultrasound not only reduces the amount of antisolvent needed to cause supersaturation and hence precipitation, but also reduces the induction times of nucleation. ${ }^{40}$ With this in mind, MacGillivray and co-workers have demonstrated that low-intensity ultrasound is capable of producing nanostructured cocrystals of resorcinol and functionalized olefins in water-ethanol which subsequently undergo crystal-to-crystal photodimerization. This transformation was only observed with small micro-sized crystals obtained under sonication; in stark contrast, cocrystals generated by reprecipitation and then irradiated with UV light did not yield the desired photoproduct and only cocrystal cracking and destruction was observed. ${ }^{41}$ Likewise, the rapid formation of caffeine-based cocrystals with a narrow size distribution, useful as APIs, has been observed after brief irradiation using antisolvent-surfactant mixtures (Fig. 6). ${ }^{42}$
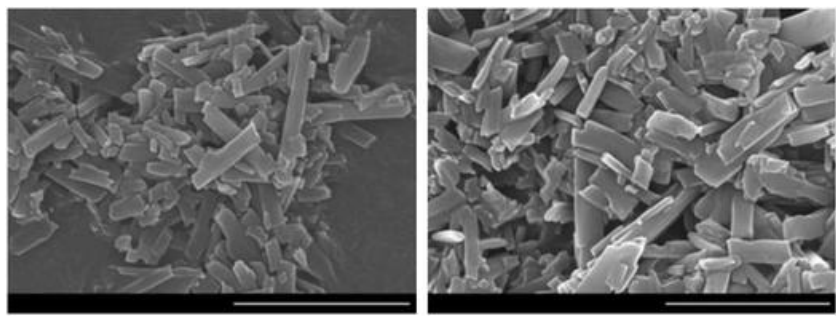

Fig. 6 SEM micrographs of agglomerated caffeine-2,4-dihydroxybenzoic acid monohydrate, (caff)·(dhba).( $\left.\mathrm{H}_{2} \mathrm{O}\right)$, using acetone and hexane as an antisolvent after ultrasonic irradiation. Scale bars are $3 \mu \mathrm{m}$ (left) and $2 \mu \mathrm{m}$ (right). Reproduced from ref. 42 by permission of Wiley-VCH Verlag $\mathrm{GmbH} \&$ Co. KGaA.

Further studies demonstrate that cocrystal formation can be achieved under sonication at higher solvent volume to sample weight ratios than when using classical liquid-assisted grinding, although the ratio also depends on the concentration of the two cocrystal components in solution. ${ }^{43}$ This parameter $(n=V / m)$ has the advantage of comparing liquid-assisted grinding with the sonochemical activation of particle slurries, with typical values between 0.1 and 1.0, which are lower than those obtained at lower saturation levels.

The same antisolvent technology is also behind the self-crystallization of $\mathrm{C}_{70}$ fullerene molecules from solution that yields crystalline cubes with strong luminescence (Fig. 7). ${ }^{44} \mathrm{C}_{70}$ powder is first dissolved in mesitylene and the subsequent addition of isopropyl alcohol induces the formation of an immiscible liquidliquid interface. The resulting precipitate however contains rectangular-shaped crystals of variable size, whereas sonication prevents random nucleation and leads to shape homogeneity. Due to the regularity of the well-defined $\mathrm{C}_{70}$ cubes obtained with this method, the crystals display enhanced photoluminescence. Moreover, the method provides $\mathrm{C}_{70}$ cube crystals that are suitable for $\mathrm{X}$-ray diffraction; the crystal structure has a simple cubic unit cell composed of one $\mathrm{C}_{70}$ and two mesitylene molecules.
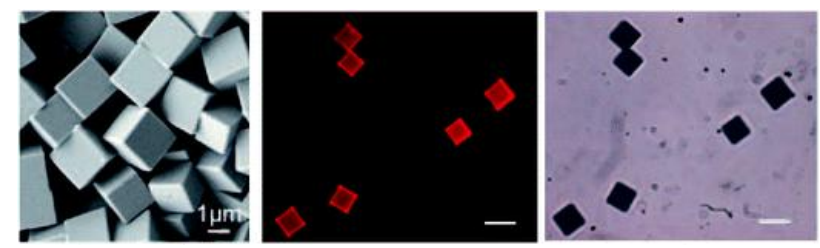

Fig. 7 High-magnification SEM image of $C_{70}$ cube crystals (left). Fluorescence optical microscope image of $C_{70}$ cubes (centre) and the corresponding transmission optical microscope image (right); the scale bars are $10 \mu \mathrm{m}$ (centre) and (right). Reproduced from ref. 44 by permission of Wiley- $\mathrm{VCH}$ 
Aizenberg et al. employed ultrasound to produce large and high-quality single crystals of mercury thiolates $\left(\mathrm{HgSR}_{2}, \mathrm{R}=\right.$ alkane), which are components of organic electronics. ${ }^{45}$ While traditional methods to make such thiolates involve the reaction of mercury salts with thiolates or disulfides and usually lead to small crystals, the present methodology combines liquid mercury and alkanethiols under a few seconds of sonication and gives rise to a rapid emulsion of $\mathrm{Hg}$ droplets in liquid alkanethiols. In fact, mercury emulsions can be generated in various solvents and were once applied to the promotion of ketone dehalogenations, one of the early examples of modern synthetic sonochemistry. ${ }^{27}$ Self-assembled monolayers (SAMs) of alkanethiols then adhere to the surface of each droplet. Sonication favours the detachment of SAMs and induces self-assembly into large crystallites. Without sonication, crystals were not obtained even after stirring for more than three hours. The process has not yet been attempted under milling or related mechanochemical-like conditions, but it could well be imagined that a fair reactivity comparison would be the use of milled reactants at the temperature employed in the ultrasonic protocol.

Accurate control of the $\mathrm{MZ}$ also enables further control of polymorphism which may have a profound influence on the physico-chemical properties of some substances. The application of ultrasound to a polymorphic system can lead to the most thermodynamically stable and least soluble polymorphs because nucleation can be attained at lower levels of supersaturation. The issue is certainly multifaceted, as revealed by a recent study on calcium carbonate polymorphs. Thus, variable results are obtained with 20 $\mathrm{kHz}$ irradiation and intensities ranging from 1.5 to $18.5 \mathrm{~W} \mathrm{~cm}^{-2}$. Short or low intensity irradiation gives rise to a high proportion of vaterite (up to $90 \%$ ), whereas longer or higher intensity irradiation (>30 min) affords $100 \%$ calcite. ${ }^{46}$ The transformation of vaterite into calcite takes place via a solution-mediated dissolutionrecrystallization process and, although sonication parameters alter the polymorphic ratio, the main factors seem to be the initial concentration of salts and the supersaturation of the solution. A subsequent study has however challenged the preferential formation of $\mathrm{CaCO}_{3}$ polymorphs as the carbonate concentration, and hence saturation levels, is dependent on the aqueous $\mathrm{CO}_{2}$ concentration, which is an often overlooked fact. The polymorph is predominantly vaterite with small amounts of calcite both with $\left(42 \mathrm{kHz}, 7.1 \mathrm{~W} \mathrm{~cm}{ }^{-2}\right)$ and without ultrasound. ${ }^{47}$ Upon monitoring $\mathrm{CaCO}_{3}$ precipitation at different time windows, it was concluded that ultrasound does not cause significant effects in the first 10 min, thus ruling out the influence of sonication on primary nucleation. Instead, ultrasound modifies precipitation during crystal growth which increases the number of crystals. The resulting increase in available surface area also leads to faster volumetric precipitation rates. The mechanical effect is chiefly attributed to shockwaves causing deaggregation and large particle breakage.

Insight into sonofragmentation mechanisms has come from another recent study by Zeiger and Suslick that deals with the irradiation of aspirin slurries using a titanium horn $(20 \mathrm{kHz}, 10 \mathrm{~W}) .{ }^{48}$ In agreement with the above study, ${ }^{47}$ a mechanism of interparticle collisions does not appear to be the principal means by which the process occurs (fragmentation is independent of slurry concentration). Moreover, experiments suggest that neither particle-horn collisions nor particle-wall collisions constitute the primary breakage source and that a direct interaction between particles and shock waves is the dominant mechanism. In addition, this result highlights the different mechanisms for the sonication of molecular crystals and metal powders (vide supra), for which interparticle collisions contribute to mechanical damage.

Current and future sonocrystallization challenges require the design of more efficient and scalable devices, operating in a continuous mode, which may have a further impact on industrial crystallization. A couple of processes, such as $\mathrm{UMAX}^{\circledR}$ (Ultrasound Mediated Amorphous to Crystalline Transition) or SAX ${ }^{\mathrm{TM}}$ (Solution 
Atomization and Crystallization by Sonication), have been introduced and applied to the production of pharmaceutical cocrystals and anti-inflammatory drugs that are usually administered by inhalation. ${ }^{36,49}$ Corticosteroids such as budesonide or fluticasone, which are employed in the treatment of respiratory diseases, are usually prepared by micronisation. However, the resulting dry powder contains large and irregular crystals. By combining spray drying and ultrasonication, the powder changes its morphology and shows a uniform size which is suitable for inhalation.

Still, the intriguing question is; how does ultrasound induce and modify nucleation? The mechanism is still uncertain because classical methods of nucleation fail or, at least, do not account for the whole phenomenon. The issue has been recently addressed in the simulation of bubble dynamics. A crucial factor appears to be convection in the medium, which is different in a sonicated system and made up of two components, namely microturbulence (or micro-convection), induced by radial motion of the cavitation bubble, and shock waves which are high-pressure waves emitted by the bubble. Shock waves increase the nucleation rate while micro-convection dictates crystal growth. The former increases by one order of magnitude with ultrasound, while growth decreases substantially with respect to a mechanically stirred reaction. ${ }^{50}$ Other studies focus on ultrasound-enhanced nucleation as a direct effect of cavitation on the agglomeration of incipient clusters. ${ }^{51}$ The model again shows that the concentration of clusters near a collapsing bubble is more than one order of magnitude higher with this technique, thus favouring aggregation and enhancing the nucleation rate.

\section{Ultrasound-assisted polymer mechanochemistry}

If any subdiscipline of covalent mechanochemistry, i.e. the application of force used to break or rearrange covalent bonds in molecular structures, has become particularly relevant in multidisciplinary chemistry, it must surely be polymer mechanochemistry. This topic has been addressed in recent reviews and readers are referred to the detailed information held therein. ${ }^{2,452,53}$ Polymer mechanochemistry not only unveils new reactivity patterns, but it is potentially relevant for activating metal-based catalysts via thermally disfavoured pathways. In addition, the control of molecular systems by mechanical stimuli provides new opportunities in varied phenomena such as molecular machines, sensors, healing, etc.

Ultrasound has long been used for both depolymerization and polymer formation. The generation of free radicals and mechanical force by the collapsing bubbles in a liquid accounts for such effects. ${ }^{52}$ In general, the degradative character of ultrasound brings to mind certain parallels with polymer mechanochemistry. Strong bonds may fragment during milling, grinding or shearing forming "mechanoradicals" with the concomitant decrease in polymer chain length. The type of cleavage is dominated by the stability of the resulting radicals which then undergo the usual pathways of addition and recombination. ${ }^{6}$

While one may easily grasp the mechanical action of shockwaves in the vicinity of solid particles, discrete molecules in solution are unlikely to experience similar effects unless bond breaking takes place via cavitation. Mechanical forces, however, may cause a polymer to elongate and put stress on its backbone. The fragmentation may also occur thermally as polymers near or at the interface of a collapsing bubble will be subjected to transient temperatures in the range of 1000-2000 K. Numerous experiments show that polymer scission under sonication is not completely stochastic in nature and occurs preferentially at the midpoint of the chain. Chemists have harnessed this selective cleavage to apply the mechanical force of ultrasound to small molecules, called mechanophores, covalently tethered to long chains on either side of a polymer backbone. So, the polymer segments behave as molecular tweezers capable of transmitting large tensile forces to small molecules in solution. 
Both the rate and extent of polymer cleavage hinge on numerous external parameters such as temperature, acoustic intensity, solvent type, dissolved gases and polymer concentration all of which influence cavitational collapse. ${ }^{2,52}$ The above considerations, that disclose the dependence of force on size, tell us that a threshold molecular weight is required for sonofragmentation to occur due to the forces arising from fluid flow after collapse. In fact, the mechanical effect of ultrasound is negligible below a critical polymer chain size.

A series of well-documented examples highlight the versatility and, in most cases, surprising reactivity possible when polymers respond to ultrasonic stress and fragment the embedded mechanophores (Scheme 2)..$^{52,53}$

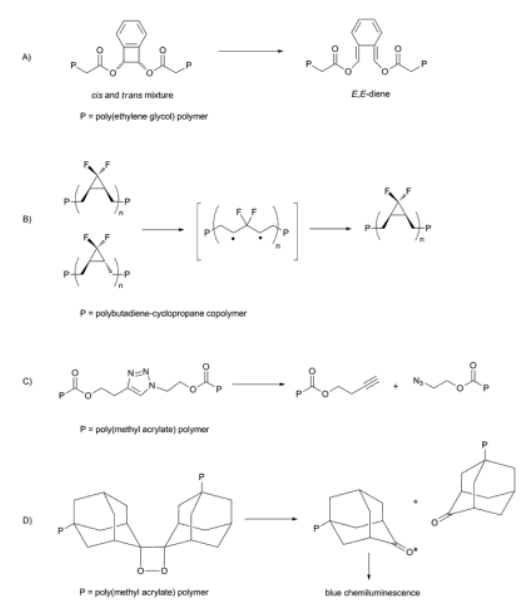

Scheme 2 Unusual chemical reactivity induced by sono-mechanochemistry.

A seminal work (A) by Moore and associates showed that the application of mechanical forces to both the cis and trans isomers of a benzocyclobutene mechanophore led to just one ring-opened diene which was in the E,E-configuration. This result goes against the tenet established by the Woodward-Hoffmann rules that predict different products for the cis and trans isomers and also disagrees with the results under thermal or photochemical activation. Apparently, sonication triggered a formally disallowed electrocyclic ring opening.

In subsequent work (B), Craig and co-workers employed a polymer containing both cis- and transdifluorocyclopropyl units. Under the action of ultrasound, the trans isomers converted into their cis counterparts, even though the trans stereochemistry is more thermodynamically stable. Pulling on the strained three-membered ring results in a diradical that can even be trapped with a nitroxide radical, which is formally a transition state of the stress-free electrocyclic isomerization. When the force ceases, the diradical returns to a point of the potential energy surface which possesses no barrier to disrotatory ring closure to the cis isomer. Overall, the trans-pulling for difluorocyclopropane follows a thermally forbidden conrotatory pathway.

Entry (C) provides another unusual transformation reported by Bielawski and his team; the application of mechanical energy using ultrasound can cyclorevert 1,2,3-triazoles into the parent alkynes and azides. This unclick reaction was not possible under the action of light or heat. Interestingly, sonication unveils the reversible character of click reactions which are usually thought to be irreversible transformations. When polymer chains that terminate in alkyne and azide groups were irradiated in the presence of $\mathrm{Cu}(\mathrm{I})$, used as a 
catalyst, reclicking took place and the resulting material had a molecular weight which was similar to the initial polymer.

The last entry (D) in Scheme 2, reported by Sijbesma and associates, ${ }^{54}$ summarizes a recent and novel application of mechanophore activation. Chemiluminescence can be mechanically induced when a bis(adamantyl)-1,2-dioxetane undergoes ring opening giving rise to two ketones, one of which is in an excited state and emits visible light. The bright-blue luminescence can be a useful diagnostic tool in checking for the failure of polymeric materials.

If mechanochemical effects under ultrasound are attracting the interest of synthetic chemists, the search for a plausible rationale behind mechanical force is an exciting new field for theorists. Some studies have been reported in recent years, although a comprehensive article by Ribas-Arino and Marx is highly recommended..$^{55}$ The authors discuss conceptual basis and computational methodologies to assess covalent mechanochemistry from a number of different viewpoints, and do not restrict themselves to sonication. Because of the unusual reaction pathways, the role exerted by ultrasound waves opens up avenues of investigation into the rules that govern stretching in an acoustic field. Theoretical calculations and molecular dynamics simulations on force-modified potential energy surfaces give insight into the changes in energy and structure of the stationary points along the reaction coordinate, although a unifying framework has not yet been established. Predictions on which system will be mechanochemically allowed or forbidden remain elusive.

If external force promotes forbidden reactions, thereby violating Woodward-Hoffmann rules for instance, changes in both electronic structure and orbital occupations may be involved. However, the mechanochemical ring opening of cyclobutenes is not due to a change in electronic structure, as would be the case in photoirradiation. Rather, the energy applied circumvents the permitted pathway via the action of two synergic effects; (1) by shifting the transition state along the intrinsic reaction coordinate toward the reactants, thus reducing the reaction barrier, and (2) by rendering the electronic factors that disfavour, for instance, diradical formation secondary, thus favouring these less common mechanisms under mechanical conditions. ${ }^{56}$

An important conclusion made by theoretical analyses is that polymer chains apparently play roles other than merely conveying mechanical force to the mechanophore. In the benzocyclobutene system, the chain length tunes the external force at which the disrotatory pathway becomes lower in energy and hence mechanochemically favoured (energy tends to decrease as the size increases). Likewise, the topology of the force-modified potential energy surface changes around the conrotatory transition state with increasing mechanical stress. The influence of the side polymer chain is especially noticeable at high external forces, for values exceeding $1.5 \mathrm{nN}$. In this case, the activation energy, as a function of the polymer chain, may vary as much as $8 \mathrm{kcal} \mathrm{mol}^{-1}$, thus biasing the reaction outcome. ${ }^{55}$ Simulations of external force using the approach known as COGEF (Constrained Geometries Simulate External Force), where the distance between two atoms is gradually increased while the energy is minimized for each step, ${ }^{57}$ have been applied to triazole unclicking. Results suggest that mechanical stretching alone is insufficient to explain the effect of ultrasound. ${ }^{58}$ The cleavage of a particular mechanophore via radical-initiated processes, solvent-assisted ring opening or the generation of excited states after bubble implosion cannot be excluded.

A future aim would entail elucidating quantitative relationships between the reaction rate and the force required to stretch the polymer (ideally based on experimental parameters such as acoustic power), as well as force-structure correlations. Still far from mathematical algorithms, preliminary contributions are worthwhile and should pave the way for further developments. Moore and associates found structure- 
mechanochemical activity relationships when cyclobutanes of variable stereochemistry and substitution degree were sonochemically cleaved in a formal [2+2] cycloaddition that produces alkenes. ${ }^{59}$ Using the above-mentioned COGEF approach, methyl esters derived from six cis and trans cyclobutanes with two, one or no cyano substituents (DCC/DCT, MCC/MCT and NCC/NCT pairs, respectively) were evaluated (Fig. 8).

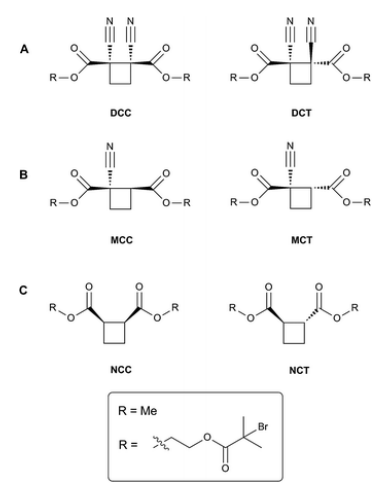

Fig. 8 (A) cis and trans Dicyano-substituted cyclobutanes (DCC and DCT), (B) cis and trans monocyano-substituted cyclobutanes (MCC and MCT), and (C) cis and trans cyclobutanes without cyano substituents (NCC and NCT). Such mechanophores were functionalized with methyl ester groups for theoretical calculations as well as with $\alpha$-bromoisobutyryl derivatives as polymerization initiators.

Theoretical calculations revealed a couple of salient conclusions; $(1)$ the cis mechanophore is more reactive than the trans isomer (i.e. less force is required to obtain bond cleavage), and (2) mechanophore reactivity increases as cyclobutane substitution increases, a fact that is attributed to enhanced ring strain caused by steric interactions. Such predictions were corroborated when the mechanophores were covalently tethered to poly(methyl acrylate) (PMA) polymers with molecular weights ranging from 35 to $125 \mathrm{kDa}$, which were grown by single-electron-transfer living-radical polymerization from the corresponding $\alpha$-bromoester initiators (Fig. 8).

Sonochemical studies were then performed on all the members of the PMA-based polymers together with two control polymers (one where the cyclobutane ring is replaced by an alkane bridge and the other a PMA homopolymer containing neither a centre-based mechanophore nor an alkane). In order to compare the relative rates of mechanophore scission under sonication, the results can be plotted as the linear relationship of $k$ (rate constant of polymer cleavage) vs. $M_{i}$ (initial average molecular weight). In agreement with the computational study, the cis derivatives were more reactive than the trans counterparts for a given substitution pattern. Likewise, the more highly-substituted mechanophores underwent faster scission than the less-highly-substituted ones (Fig. 9). The control polymers were less reactive under ultrasound than those containing the cyclobutane ring with the sole exception of the NCT mechanophore.

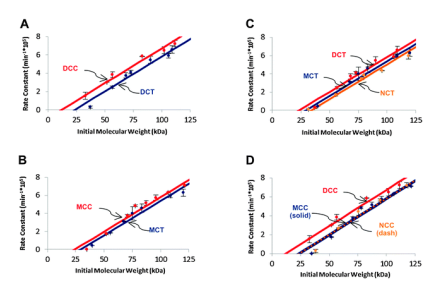

Fig. 9 Plots showing linear relationships between experimental ultrasound-assisted polymer scission rate constants and initial polymer molecular weights for a series of cyclobutane-containing chains: $(A)$ cleavage of DCC $\left(R^{2}=0.974\right)$ and $D C T\left(R^{2}=0.962\right)$ polymers, $(B)$ cleavage of MCC $\left(R^{2}=\right.$ $0.958)$ and MCT $\left(R^{2}=0961\right)$ polymers, $(C)$ cleavage of DCT, MCT and NCT polymers, and (D) cleavage of DCC, MCC and NCC polymers. Adapted and 
In a recent study, Boulatov et al. demonstrated that force-reactivity measurements in a single mechanophore (gem-dibromocyclopropane) are sufficient to predict the micromechanical behaviour of a polymer chain composed of thousands of connected dibromocyclopropanes with considerable accuracy. ${ }^{60}$ The authors carried out both experimental and extensive computational calculations on a series of cyclopropane derivatives, including strained macrocycles (Fig. 10). These results are an additional significant achievement in the continuing refining of a conceptual basis for chemomechanics, as a physical property (force) that can be quantified defines the dynamic behaviour of cyclopropanated polymers across length scales between $<1 \mathrm{~nm}$ and $>100 \mathrm{~nm}$. Obviously, this scenario may not be general, or extrapolated to other mechanophores, and may not take into account the peculiarities of some force fields, especially ultrasonic waves where bubble dynamics constitutes a complex issue. However, all these results collectively constitute a significant step forward in the search for chemomechanical relationships in molecules of different sizes.

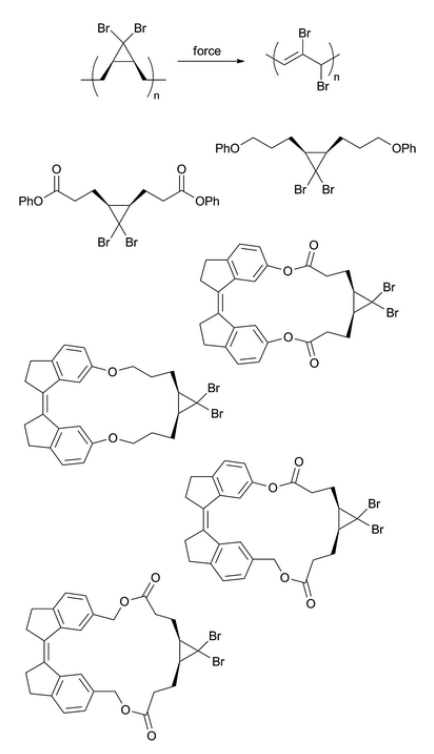

Fig. 10 gem-Dibromocyclopropanes employed in chemical and computational studies of force-induced cleavage to give alkenes.

\section{Conclusions}

Sound, pressure waves propagating through an elastic medium, is a clear-cut manifestation of mechanical energy. Sound at high frequencies (ultrasound) and at different power scales can be used for a variety of purposes in applied chemistry. Historically, low-energy standing waves have proven their mechanical action and usefulness in separation technology. Such vibrations have found a niche in the development of lab-ona-chip platforms and can induce chemical transformations, particularly on the microscale. When cavitation is present, ultrasound waves provide a unique scenario of chemomechanical energy transmission. In close mimicry of mechanochemical milling, in its production of sparkling plasmas and radicals by covalent bond breaking, cavitation induces the formation of radicals and excited states in solution along with large shockwaves and shear forces that result from microbubble implosion. Such forces affect the morphology of solids and change their physicochemical properties. Along with classical domains of sonochemistry involving inorganic solids and metals, the emerging area of sonocrystallization reveals key advantages in terms of fast nucleation and size control, which are suitable for the pharmaceutical industry. Finally, small 
molecules can be pulled open mechanically with the aid of ultrasound-induced polymer scission, and is an area which holds great promise in innovative and green chemistry. Clearly, sonication enables chemistry that is simply not possible any other way.

Mechanical activation by ultrasound is a familiar concept among sonochemists. However, both its frequent practitioners and those entering the field to harness its potential must recognize that this force depends largely on parameters and effects that result from acoustic cavitation. In general, output power, solvent and external temperature may play a decisive role in sonochemical reactivity. In addition, some data suggest that an inverse dependence exists between mechanical and chemical effects on ultrasound frequency. A Socratic golden rule is quite pertinent here: know thy sound field. The body of research in sonochemistry can be used as a source of case studies and a basis for discussion, learning and the continuing cultivation of the potentiality of this fascinating discipline.

\section{Acknowledgements}

We thank Dr. David Fernandez Rivas for his technical assistance. This work was funded by the Spanish Ministerio de Ciencia e Innovación (Grant CTQ2010-18938/BQU), the Junta de Extremadura-Fondos FEDER (Ayuda a Grupos Consolidados GR10049), and by the Compagnia di San Paolo - University of Turin (progetti di ricerca di Ateneo 2011, grant D15E11001710003).

\section{Notes and references}

1. Z. V. Todres, Organic Mechanochemistry and Its Practical Applications, CRC Press Inc., Boca Raton, FL, 2006.

2. M. M. Caruso, D. A. Davis, Q. Shen, S. A. Odom, N. R. Sottos, S. R. White and J. S. Moore, Chem. Rev., 2009, 109, 5755-5798.

3. Z. Huang and R. Boulatov, Chem. Soc. Rev., 2011, 40, 2359-2384.

4. C. Weder (ed.), Special issue on mechanoresponsive materials, J. Mater. Chem., 2011, 21, 82178476 .

5. S. L. James, C. J. Adams, C. Bolm, D. Braga, P. Collier, T. Friščić, F. Grepioni, K. D. M. Harris, G. Hyett, W. Jones, A. Krebs, J. Mack, L. Maini, A. G. Orpen, I. P. Parkin, W. C. Shearouse, J. W. Steed and D. C. Waddell, Chem. Soc. Rev., 2012, 41, 413-447.

6. G. Kaupp, in Making Crystals by Design, ed. D. Braga and F. Grepioni, Wiley-VCH, New York, 2007, pp. 87-148 ; G. Kaupp, J. Phys. Org. Chem., 2008, 21, 630-643 ; G. Kaupp, CrystEngComm, 2009, $11,388-403$.

7. G. Cravotto and P. Cintas, Chem. Soc. Rev., 2006, 35, 180-196 .

8. The New Encyclopaedia Britannica, Enc. Britannica Inc., Chicago, 15th edn, 1990, vol. 27, pp. 604632 .

9. T. J. Mason and J. P. Lorimer, Applied Sonochemistry. The Uses of Power Ultrasound in Chemistry and Processing, Wiley-VCH, Weinheim, 2002, pp. 1-24 .

10. M. Faraday, Philos. Trans. R. Soc. London, 1831, 121, 299-318 (app. 318-340). 
11. R. Hoyle, Pattern Formation. An Introduction to Methods, Cambridge University Press, Cambridge, 2006, pp. 20-22 .

12. A. Kundt, Ann. Phys., 1866, 127, 497-523 .

13. L. Matthiessen, Ann. Phys., 1868, 210, 107-117.

14. K. Sollner and C. Bondy, Trans. Faraday Soc., 1936, 32, 616-623 .

15. M. Dyson, B. Woodward and J. B. Pond, Nature, 1971, 232, 572-573 .

16. T. J. Mason, Ultrason. Sonochem., 2011, 18, 847-852 .

17. D. Mark, S. Haeberle, G. Roth, F. von Stetten and R. Zengerle, Chem. Soc. Rev., 2010, 39, 11531182 .

18. Z. Wang and J. Zhe, Lab Chip, 2011, 11, 1280-1285 .

19. K. Kulkarni, J. Friend, L. Yeo and P. Perlmutter, Lab Chip, 2009, 9, 754-755 .

20. J.-L. Luche, C. Einhorn, J. Einhorn and J. V. Sinisterra-Gago, Tetrahedron Lett., 1990, 31, 41254128 .

21. L. A. Crum, Ultrason. Sonochem., 1995, 2, S147-S152 .

22. W. Ostwald, Handbuch der allgemeine Chemie, Band I, Academische Verlagsgesellchaft, Leipzig, 1919, p. 70 .

23. P. Baláž, Mechanochemistry in Nanoscience and Minerals Engineering, Springer, Berlin, 2008, ch. 1, pp. 1-29 .

24. N. K. Goh, A. C. C. Teoh, L. S. Chia and K. C. Teo, Ultrason. Sonochem., 1996, 3, S209-S214 .

25. F. P. Bowden and A. D. Yoffe, Initiation and Growth of Explosion in Liquids and Solids, Cambridge University Press, Cambridge, 1952, p. 89 .

26. V. V. Boldyrev, Ultrason. Sonochem., 1995, 2, S143-S145 .

27. P. Cintas and J.-L. Luche, in Synthetic Organic Sonochemistry, ed. J.-L. Luche, Plenum Press, New York, 1998, pp. 167-234 .

28. P. Cintas, G. Palmisano and G. Cravotto, Ultrason. Sonochem., 2011, 18, 836-841 .

29. P. Cintas, A. Barge, T. Tagliapietra, L. Boffa and G. Cravotto, Nat. Protocols, 2010, 5, 607-616 .

30. D. G. Shchukin, E. Skorb, V. Belova and H. Möhwald, Adv. Mater., 2011, 23, 1922-1934 .

31. S. Verdan, G. Burato, M. Comet, L. Reinert and H. Fuzellier, Ultrason. Sonochem., 2003, 10, 291295.

32. A. Brotchie, D. Borisova, V. Belova, H. Möhwald and D. Shchukin, J. Phys. Chem. C, 2012, 116, 79527956 . 
33. E. V. Skorb, D. Fix, D. G. Shchukin, H. Möhwald, D. V. Sviridov, R. Mousa, N. Wanderka, J. Schäferhans, N. Pazos-Pérez, A. Fery and D. V. Andreeva, Nanoscale, 2011, 3, 985-993 .

34. Y. Wang, D. Zhao, W. Ma, C. Chen and J. Zhao, Environ. Sci. Technol., 2008, 42, 6173-6178 .

35. Y.-H. Lee, G. Kim, M. Joe, J.-H. Jang, J. Kim, K.-R. Lee and Y.-U. Kwon, Chem. Commun., 2010, 46, 5656-5658.

36. G. Ruecroft, D. Hipkiss, T. Ly, N. Maxted and P. W. Cains, Org. Process Res. Dev., 2005, 9, 923-932 .

37. O. Narducci, A. G. Jones and E. Kougoulos, Cryst. Growth Des., 2011, 11, 1742-1749 .

38. S. James and A. Nangia (ed.), Mechanochemistry and co-crystals (special issue), CrystEngComm, 2009, 11, 375-522.

39. S. L. Childs and M. J. Zaworotko (ed.), Virtual issue on pharmaceutical co-crystals, Cryst. Growth. Des., 2009, vi, 1. Links to all the articles viahttp://pubs.acs.org/page/cgdefu/vi/1 (accessed: October 2012).

40. E. Revalor, Z. Hammadi, J.-P. Astier, R. Grossier, E. Garcia, C. Hoff, K. Furuta, T. Okustu, R. Morin and S. Veesler, J. Cryst. Growth, 2010, 312, 939-946.

41. D.-K. Bučar and L. R. MacGillivray, J. Am. Chem. Soc., 2007, 129, 32-33 .

42. J. R. G. Sander, D.-K. Bučar, R. F. Henry, G. G. Zhang and L. R. MacGillivray, Angew. Chem., Int. Ed., 2010, 49, 7284-7288.

43. T. Friščić, S. L. Childs, S. A. A. Rizvi and W. Jones, CrystEngComm, 2009, 11, 418-426.

44. C. Park, E. Yoon, M. Kawano, T. Joo and H. C. Choi, Angew. Chem., Int. Ed., 2010, 49, 9670-9675 .

45. B. Pokroy, B. Aichmayer, A. S. Schenk, B. Haimov, S. H. Kang, P. Fratzl and J. Aizenberg, J. Am. Chem. Soc., 2010, 132, 14355-14357 .

46. G. J. Price, M. F. Mahon, J. Shannon and C. Cooper, Cryst. Growth Des., 2011, 11, 39-44 .

47. R. M. Wagterveld, H. Miedema and G.-J. Witkamp, Cryst. Growth Des., 2012, 12, 4403-4410 .

48. B. W. Zeiger and K. S. Suslick, J. Am. Chem. Soc., 2011, 133, 14530-14533 .

49. G. Ruecroft, D. Parikh and D. Hipkiss, World Patent, WO 10/007447, 2010 .

50. V. S. Nalajala and V. S. Moholkar, Ultrason. Sonochem., 2011, 18, 345-355 .

51. H. Harzali, F. Baillon, O. Louisnard, F. Espitalier and A. Mgaidi, Ultrason. Sonochem., 2011, 18, 1097-1106.

52. G. Cravotto and P. Cintas, Chem. Sci., 2012, 3, 295-307.

53. K. M. Wiggins, J. N. Brantley and C. W. Bielawski, ACS Macro Lett., 2012, 1, 623-626 .

54. Y. Chen, A. J. H. Spiering, S. Karthikeyan, G. W. M. Peters, E. W. Meijer and R. P. Sijbesma, Nat. Chem., 2012, 4, 559-562 . 
55. J. Ribas-Arino and D. Marx, Chem. Rev., 2012, 112, 5412-5487.

56. G. S. Kochhar, A. Bailey and N. J. Mosey, Angew. Chem., Int. Ed., 2010, 49, 7452-7455.

57. M. K. Beyer, J. Chem. Phys., 2000, 112, 7307-7312.

58. H. S. Smalø and E. Uggerud, Chem. Commun., 2012, 48, 10443-10445.

59. M. J. Kryger, A. M. Munaretto and J. S. Moore, J. Am. Chem. Soc., 2011, 133, 18992-18998.

60. S. Akbulatov, Y. Tian and R. Boulatov, J. Am. Chem. Soc., 2012, 134, 7620-7623. 